Discrete Comput Geom 35:537-549 (2006)

DOI: $10.1007 / \mathrm{s} 00454-006-1232-4$

\title{
Distinct Distances in Homogeneous Sets in Euclidean Space*
}

\author{
József Solymosi ${ }^{1}$ and Csaba D. Tóth ${ }^{2}$ \\ ${ }^{1}$ Department of Mathematics, University of British Columbia, \\ Vancouver, British Columbia, Canada V6T 1Z2 \\ solymosi@math.ubc.ca \\ ${ }^{2}$ Department of Mathematics, Massachusetts Institute of Technology, \\ Cambridge, MA 02139, USA \\ toth@math.mit.edu
}

\begin{abstract}
It is shown that every homogeneous set of $n$ points in $d$-dimensional Euclidean space determines at least $\Omega\left(n^{2 d /\left(d^{2}+1\right)} / \log ^{c(d)} n\right)$ distinct distances for a constant $c(d)>0$. In three-space the above general bound is slightly improved and it is shown that every homogeneous set of $n$ points determines at least $\Omega\left(n^{0.6091}\right)$ distinct distances.
\end{abstract}

\section{Introduction}

The history of the distinct distance problem goes back to Erdôs [10] who asked the question: What is the minimal number $g_{d}(n)$ of distinct distances determined by $n$ points in $d$-dimensional Euclidean space $\mathbb{R}^{d}$ ? $n$ points in the $d$-dimensional integer grid $\left[1,2, \ldots, n^{1 / d}\right]^{d}$ show that $g_{d}(n)=O\left(n^{2 / d}\right)$ for any $d \geq 2$ and, in particular, $g_{2}(n)=O(n / \sqrt{\log n})$. Erdôs conjectured that these bounds are essentially optimal [11].

An initial lower bound of $g_{2}(n) \geq \Omega(\sqrt{n})$ by Erdős [10] was improved over the last almost 60 years by Moser, Beck, Chung, Szemerédi, Trotter, and Székely [19], [3], [5], [6], [25]. Research efforts have lead to several powerful methods (such as the crossing theory [25] and the $\varepsilon$-cutting theory [7]) which, in turn, found innumerable applications in discrete and computational geometry. An excellent survey by Pach and Sharir [20] elaborate on the history of the distinct distance problem and its connections to other fields of discrete mathematics. Determining the order of magnitude of $g_{2}(n)$ (and $g_{d}(n)$ for every $d \in \mathbb{N}$ ) seems elusive. The currently known best lower bound in the plane,

* The research by József Solymosi was supported by OTKA and NSERC grants. 
$g_{2}(n)=\Omega\left(n^{0.8641}\right)$, is due to Katz and Tardos [17]. Their proof combines a method of Solymosi and Tóth [21] with results from entropy and additive number theory.

Not much work has been done in higher dimensions. After some initial results by Clarkson et al. [7] and by Spencer et al. [24], Aronov et al. [2] have showed recently that the number of distinct distances determined by a set of $n$ points in three-dimensional space is $g_{3}(n)=\Omega\left(n^{77 / 141-\varepsilon}\right)=\Omega\left(n^{0.5460}\right)$ for any $\varepsilon>0$. Solymosi and $\mathrm{Vu}$ [23] proved a general lower bound of $g_{d}(n)=\Omega\left(n^{2 / d-2 / d(d+2)}\right)$ for any fixed $d \geq 4$.

In this paper we consider the minimum number $h_{d}(n)$ of distinct distances in homogeneous sets of $n$ points in $\mathbb{R}^{d}$. A finite point set $P \subset \mathbb{R}^{d}$ is homogeneous if the following two conditions hold: $P$ lies in the interior of an axis-aligned $d$-dimensional cube $C$ of volume $|P|$, and any unit cube in $\mathbb{R}^{d}$ contains at most $O(1)$ points of $P$. Homogeneous sets represent an important special case for the distinct distance problem because the best known upper bound constructions (the $d$-dimensional integer grids) are homogeneous, and because of numerous connections to harmonic analysis [4], [12], [14], [16], [18]. Iosevich [13] studied the distinct distance problem for homogeneous sets (with additional restrictions). He showed that $h_{d}(n)=\Omega\left(n^{3 / 2 d}\right)$ for any fixed $d \geq 2$. Solymosi and $\mathrm{Vu}$ [22] proved a general bound of $h_{d}(n)=\Omega\left(n^{2 / d-1 / d^{2}}\right)$ for every dimension $d \geq 2$. For $d=3$, they have also obtained a slightly better bound $h_{3}(n)=\Omega\left(n^{0.5794}\right)$. In this paper we improve all previous lower bounds on the number of distinct distances in homogeneous sets of $n$ points in $\mathbb{R}^{d}, d \geq 3$.

Theorem 1. For every $d \in \mathbb{N}$, there is a constant $c_{d}$ such that in every homogeneous set $P$ of $n$ points in $\mathbb{R}^{d}$, there is a point $p \in P$ from which there are at least

$$
c_{d} n^{2 d /\left(d^{2}+1\right)} \log ^{\left(1-d^{2}\right) /\left(d^{2}+1\right)} n
$$

distinct distances measured to other points of $P$. In particular, we have $h_{d}(n) \geq$ $c_{d} n^{2 d /\left(d^{2}+1\right)} \log ^{\left(1-d^{2}\right) /\left(d^{2}+1\right)} n$.

For $d=3,4$, and 5, our general lower bound is $h_{3}(n)=\Omega\left(n^{0.5999}\right), h_{4}(n)=$ $\Omega\left(n^{0.4705}\right)$, and $h_{5}(n)=\Omega\left(n^{0.3846}\right)$. In three dimensions we slightly improve on this bound and prove the following:

Theorem 2. In every homogeneous set $P$ of $n$ points in $\mathbb{R}^{3}$, there is a point $p \in P$ from which there are at least

$$
\Omega\left(n^{53 / 87}\right)=\Omega\left(n^{0.6091}\right)
$$

distinct distances measured to other points of $P$. In particular, we have $h_{3}(n)=$ $\Omega\left(n^{53 / 87}\right)$.

We prove Theorem 1 in Section 3. The proof of Theorem 2 can be found in Section 4. In the next section we present a key lemma on the number of $k$-flats incident to many points in a homogeneous point set in $\mathbb{R}^{d}$, for $1 \leq k<d$. 


\section{Rich Hyperplanes in Homogeneous Sets}

Consider a set $P$ of $n$ points in $\mathbb{R}^{d}$. We say that a $k$-flat (a $k$-dimensional affine subspace) is $m$-rich if it is incident to at least $m$ points of $P$. The celebrated Szemerédi-Trotter Theorem [26] states that for $n$ points in the plane, the number of $m$-rich lines (1-flats) is at most $O\left(n^{2} / m^{3}+n / m\right)$, and this bound is tight in the worst case.

The number of $m$-rich $k$-flats in $\mathbb{R}^{d}$ has been intensely studied. The SzemerédiTrotter type results have widespread applications in discrete and combinatorial geometry. The Szemerédi-Trotter Theorem's multi-dimensional generalizations [1], [8], [9] always impose some kind of restriction on the point set or on the set of $k$-flats, otherwise $m$ points on a line give rise to infinitely many $m$-rich $k$-flats for any $2 \leq k \leq d$.

We adopt the following terminology. A set of $k+1$ points in $\mathbb{R}^{d}, k \leq d$, is affine independent if it is contained in a unique $k$-flat, which is said to be spanned by the point set. A point set $P$ determines all the $k$-flats spanned by some $k+1$ affine independent points of $P$. For a constant $\alpha>0$, a finite point set $P \subset \mathbb{R}^{d}$ that spans a $k$-flat is $\alpha$-degenerate if any $(k-1)$-flat contains at most $\alpha \cdot|P|$ points of $P$. For a finite point set $P \subset \mathbb{R}^{d}$ and a constant $\alpha>0$, we say that a $k$-flat $F$ is $\alpha$-degenerate if the point set $P \cap F$ is $\alpha$-degenerate. Note, for example, that all points of $P \cap F$ in a 1-degenerate $k$-flat $F$ may lie on a $(k-1)$-flat, but an $\alpha$-degenerate $k$-flat for $\alpha<1$ must be spanned by points of $P$. We recall a result of Beck [3] on $\alpha$-degenerate hyperplanes.

Theorem 3 [3]. For every $k \in \mathbb{N}$, there are constants $\alpha_{k}, \beta_{k}>0$ with the following property. For everyd $\in \mathbb{N}$ and every finite point set $P \subset \mathbb{R}^{d}$, if a $k$-flat $F$ is $\alpha_{k}$-degenerate, then $P \cap F$ spans at least $\beta_{k} \cdot|F \cap P|^{k}$ distinct $(k-1)$-flats.

Elekes and Tóth [9] proved that for every dimension $d \in \mathbb{N}$, there is a constant $\gamma_{d}>0$ such that the number of $m$-rich $\gamma_{d}$-degenerate hyperplanes for $n$ points in $\mathbb{R}^{d}$ is at most $O\left(n^{d} / m^{d+1}+n^{d-1} / m^{d-1}\right)$. The first term, $O\left(n^{d} / m^{d+1}\right)$, is dominant only if $m=O(\sqrt{n})$. We show below a much stronger upper bound for homogeneous sets. A homogeneous set of $n$ points in $\mathbb{R}^{d}$ determines at most $O\left(n^{d} / m^{d+1}\right)$ distinct $m$-rich hyperplanes for every $m \in \mathbb{N}, d \leq m \leq n$.

We formulate our result for a slightly more general class of point sets, where $n$ denotes the volume of the enclosing cube, rather than the number of points. We say that a point set $P$ is well separated if any unit cube in $\mathbb{R}^{d}$ contains at most $O(1)$ points of $P$. By definition, every homogeneous set of $n$ points in $\mathbb{R}^{d}$ is well separated, and lies in a cube of volume $n$.

Let $f_{d, k}(P, m)$ denote the maximal number of $m$-rich $k$-flats in a well separated point set $P$ contained in the interior of a $d$-dimensional cube of volume $n$ in $\mathbb{R}^{d}$, and let

$$
f_{d, k}(n, m)=\max _{P \subset \mathbb{R}^{d},|P|=n} f_{d, k}(P, m) .
$$

Solymosi and $\mathrm{Vu}$ [22] established the following lemma for the number of $m$-rich lines in homogeneous sets of $n$ points in $\mathbb{R}^{d}$. Their proof carries over verbatim for well separated sets of volume $n$. 
Lemma 4 [22]. For every $d \in \mathbb{N}$, there is a constant $c_{d}$ such that

$$
f_{d, 1}(n, m) \leq c_{d} \frac{n^{2}}{m^{d+1}}
$$

We extend their result for arbitrary $k \in \mathbb{N}, 1 \leq k \leq d-1$.

Lemma 5. For every $d, k \in \mathbb{N}, 1 \leq k<d$, there is a constant $c_{d, k}$ such that

$$
f_{d, k}(n, m) \leq c_{d, k} \frac{n^{k+1}}{m^{d+1}} .
$$

The example of the $d$-dimensional integer grid $\left[1,2, \ldots, n^{1 / d}\right]^{d}$ shows that this bound is best possible for every $m \in \mathbb{N}, 1 \leq m \leq n^{k / d}$.

Proof. For a fixed $d \in \mathbb{N}$, we prove that $f_{d, k}(n, m)=O\left(n^{k+1} / m^{d+1}\right)$. We proceed by induction on $k, 1 \leq k \leq d$. The base case, $k=1$, is equivalent to Lemma 4 . Assume that $1<k \leq d$ and that $f_{d, k_{0}}\left(n_{0}, m\right)=O\left(n_{0}^{k_{0}+1} / m^{d+1}\right)$ for every $k_{0}, 1 \leq k_{0}<k$, and $n_{0} \in \mathbb{N}$.

Consider a well separated set $P$ that lies in the interior of a $d$-dimensional cube $C$ of volume $n$. Clearly, we have $|P|=O(n)$. We may choose an orthogonal coordinate system such that all coordinates of every point of $P$ are irrational and $P$ lies in the interior of cube $C$, whose vertices have rational coordinates. This guarantees that for any subdivision of $C$ into congruent subcubes, every point of $P$ lies in the interior of a subcube. For $i=0,1, \ldots,\left\lceil\log n^{1 / d}\right\rceil$, let $\mathcal{C}_{i}$ denote the subdivision of the cube $C$ into $2^{i d}$ congruent cubes. For instance, $\mathcal{C}_{0}=\{C\}, \mathcal{C}_{1}$ is a subdivision of $C$ into $2^{d}$ cubes, and $\mathcal{C}_{\lceil(\log n) / d\rceil}$ is a subdivision into constant volume cubes. There is a constant $\delta_{d}>d$ such that every $k$-flat $F$ intersects at most $\delta_{d}\left|\mathcal{C}_{i}\right|^{k / d}=\delta_{d} 2^{i k}$ cubes of $\mathcal{C}_{i}$. If we put

$$
\mu=\left\lfloor\frac{1}{k} \log \frac{m}{4 \delta_{d}(k+1)}\right\rfloor
$$

then every $m$-rich $k$-flat $F$ is incident to an average of at least $m /\left(\delta_{d} 2^{\mu k}\right) \geq 4(k+1)$ points in a cube $Q \in C_{\mu}$. That is, at least $m / 2$ points of $P \cap F$ lie in subcubes $Q \in C_{\mu}$ where $|P \cap F \cap Q| \geq 2(k+1)$.

Let $\alpha_{k}$ and $\beta_{k}$ be the constants from Theorem 3. Let $\mathcal{F}$ denote the $m$-rich $k$-flats. We classify the $k$-flats in $\mathcal{F}$ as follows:

- $\mathcal{F}_{1}=\left\{F \in \mathcal{F}: P \cap F\right.$ is not $\alpha_{k}$-degenerate $\}$

- $\mathcal{F}_{2}=\left\{F \in \mathcal{F}\right.$ : at least $m / 4$ points of $P \cap F$ lie in cubes $Q \in C_{\mu}$ such that the point set $P \cap F \cap Q$ is $\alpha_{k}$-degenerate $\}$,

- $\mathcal{F}_{3}=\mathcal{F} \backslash\left(\mathcal{F}_{1} \cup \mathcal{F}_{2}\right)$.

We show below that $\left|\mathcal{F}_{q}\right|=O\left(n^{k+1} / m^{d+1}\right)$, for $q=1,2$, and 3 . Every $F \in \mathcal{F}_{1}$ contains an $\left(\alpha_{k} m\right)$-rich $(k-1)$-flat. By induction, the number of $\left(\alpha_{k} m\right)$-rich $(k-1)$-flats is $O\left(n^{k} /\left(\alpha_{k} m\right)^{d+1}\right)=O\left(n^{k} / m^{d+1}\right)$. Every $\left(\alpha_{k} m\right)$-rich $(k-1)$-flat $R$ can be extended to an $m$-rich $k$-flat in $O(n)$ different ways: $R$ together with a point of $P \backslash R$ spans a $k$-flat. This gives an upper bound $\left|\mathcal{F}_{1}\right|=O\left(n^{k+1} / m^{d+1}\right)$. 
For an upper bound on $\left|\mathcal{F}_{2}\right|$, we consider the subdivision $\mathcal{C}_{\mu}$. Let $K$ denote the affine independent $(k+1)$-element subsets of $P$ that determine some $m$-rich $k$-flat in $\mathcal{F}_{2}$ and lie in some cube $Q \in \mathcal{C}_{\mu}$. The volume of every cube $Q \in \mathcal{C}_{\mu}$ is $O\left(n / 2^{\mu d}\right)=O\left(n / m^{d / k}\right)$. Since $P$ is well separated, we have $|P \cap Q|=O\left(n / m^{d / k}\right)$. A trivial upper bound for the number of affine independent $(k+1)$-element sets in all cubes of $\mathcal{C}_{\mu}$ is

$$
|K| \leq\left|\mathcal{C}_{\mu}\right| \cdot\left(O\left(\frac{n}{m^{d / k}}\right)\right)^{k+1}=O\left(\frac{n^{k+1}}{m^{d}}\right) .
$$

We obtain a lower bound for $|K|$ by counting the affine independent sets in each $F \in \mathcal{F}_{2}$. At least $m / 4$ points of $P \cap F$ lie in cubes $Q \in \mathcal{C}_{\mu}$ where the point set $P \cap F \cap Q$ is $\alpha_{k}$-degenerate. By Theorem 3, every $\alpha_{k}$-degenerate set $P \cap F \cap Q$ determines at least $\beta_{k}|P \cap F \cap Q|^{k+1}$ affine independent $(k+1)$-element sets. If we denote by $K(F)$ the number of $(k+1)$-element subsets of $K$ that span $F$, then we have

$$
|K(F)| \geq \sum_{\substack{Q \in \mathcal{C}_{\mu} \\ Q \cap F \neq \emptyset}} \beta_{k}|P \cap F \cap Q|^{k+1} \geq \delta_{d} 2^{\mu k}\left(\frac{m / 4}{\delta_{d} 2^{\mu k}}\right)^{k+1}=\Omega\left(m^{k+1} 2^{-\mu k^{2}}\right)=\Omega(m) .
$$

We conclude that $|K|=\sum_{F \in \mathcal{F}_{2}} \Omega(m)=\left|\mathcal{F}_{2}\right| \cdot \Omega(m)$. By contrasting the upper and lower bounds for $|K|$, we get $\left|\mathcal{F}_{2}\right|=O\left(n^{k+1} / m^{d+1}\right)$.

Finally, we consider $\mathcal{F}_{3}$. For every $m$-rich $k$-flat $F \in \mathcal{F}_{3}$, we define a set $S(F)$ of cubes from $\mathcal{C}_{i}, i=1,2, \ldots, \log n^{1 / d}$. A cube $Q \in \mathcal{C}_{i}$ is in $S(F)$ if and only if the point set $P \cap F \cap Q$ is not $\alpha_{k}$-degenerate, but $P \cap F \cap Q\left(i^{\prime}\right)$ is $\alpha_{k}$-degenerate for every $i^{\prime}$, $0 \leq i^{\prime}<i$, where $Q\left(i^{\prime}\right)$ is the (unique) cube $Q\left(i^{\prime}\right) \in C_{i^{\prime}}$ containing $Q$. If $P \cap F$ is not $\alpha_{k}$-degenerate, for example, then $C \notin S(F)$. Observe that the cubes of $S(F)$ are pairwise interior disjoint and they jointly cover $P \cap F \cap C$. We denote by $\operatorname{dim}(X)$ the dimension of the affine subspace spanned by a finite point set $X$. For each $F \in F_{3}$, we further classify the cubes in $S(F)$ according to three parameters: For $i \in\{1,2, \ldots, \mu\}$, $j \in\{0,1, \ldots, \log m\}$, and $r \in\{1, \ldots, k-1\}$, let $S(F, i, j, r)$ denote the set of cubes $Q \in S(F)$ such that

1. $Q \in \mathcal{C}_{i}$,

2. $2^{j-1} \cdot m / \delta_{d} 2^{i k} \leq|P \cap F \cap Q|<2^{j} \cdot m / \delta_{d} 2^{i k}$,

3. $r=\min (k-1, \operatorname{dim}(P \cap F \cap Q))$.

Some of the cubes $Q \in S(F)$ are not included in any $S(F, i, j, r,) \subset S(F)$ : This is the case for every $Q \in S(F) \cap \mathcal{C}_{i}$ for which $|P \cap F \cap Q|<\left(m / \delta_{d} 2^{i k+1}\right)$ or $\mu<i$. The total of number points of $P \cap F$ in these cubes is less than

$$
\sum_{\substack{Q \in S(F) \cap \mathcal{C}_{i} \\ 0<i<\mu}}|P \cap F \cap Q|+\sum_{\substack{Q \in S(F) \cap \mathcal{C}_{i} \\ i \geq \mu}} \frac{m}{\delta_{d} 2^{i k}}<\frac{m}{2}+\frac{m}{4}=\frac{3 m}{4} .
$$

Therefore, the cubes in $S(F, i, j, r)$ for all $i, j, r$ jointly contain at least $m / 4$ points of $P \cap F:$

$$
\sum_{i=1}^{\mu} \sum_{j=0}^{\log m} \sum_{r=1}^{k-1}|S(F, i, j, r)| \cdot \frac{2^{j-1} m}{\delta_{d} 2^{i k}} \geq \frac{m}{4}
$$


For every $Q \in S(F, i, j, r)$, there is an $r$-flat $R \subset F$, such that $|P \cap R \cap Q| \geq$ $\alpha_{k}|P \cap F \cap Q| \geq \alpha_{k} 2^{j-1} m /\left(\delta_{d} 2^{i k}\right)=\Theta\left(2^{j-i k} m\right)$. Let us denote by $Q^{\prime}$ the cube in $\mathcal{C}_{i-1}$ that contains $Q \in \mathcal{C}_{i}$. Since $P \cap F \cap Q^{\prime}$ is already $\alpha_{k}$-degenerate, we have $|P \cap R \cap Q| \leq \alpha_{k}\left|P \cap F \cap Q^{\prime}\right|$. Let $D(Q, R)$ be the set of all $(k-r)$-element affine independent sets $u \subset\left(P \cap F \cap Q^{\prime}\right) \backslash R$ such that $R$ and $u$ together span $F$. Since $P \cap F \cap Q^{\prime}$ is $\alpha_{k}$-degenerate, there are $\Theta\left(\left|P \cap F \cap Q^{\prime}\right|^{k-r}\right)$ sets in $D(Q, R)$. Let $D^{\prime}(Q, R)$ be a subset of $D(Q, R)$ of size $\Theta\left(|P \cap F \cap Q|^{k-r}\right)=\Theta\left(\left(m 2^{j-i k}\right)^{k-r}\right)$.

Let $T(F, i, j, r)$ denote the set of triples $(Q, R, u)$ such that $Q \in S(F, i, j, r), R$ is an $r$-flat with $|P \cap R \cap Q| \geq \alpha_{k}|P \cap F \cap Q|$, and $u \in D^{\prime}(Q, R)$. We have a lower bound

$$
|T(F, i, j, r)| \geq|S(F, i, j, r)| \cdot \Theta\left(\left(m 2^{j-i k}\right)^{k-r}\right) .
$$

Let us put

$$
\tau(F, i, j, r)=\frac{|T(F, i, j, r)|}{\left(m 2^{j-i k}\right)^{k-r-1}},
$$

and then inequality (1) can be rewritten as

$$
\sum_{i=1}^{\mu} \sum_{j=0}^{\log m} \sum_{r=1}^{k-1} \tau(F, i, j, r) \geq \sum_{i=1}^{\mu} \sum_{j=0}^{\log m} \sum_{r=1}^{k-1}|S(F, i, j, r)| \cdot \Omega\left(m 2^{j-i k}\right) \geq \Omega(m) .
$$

By summing over all $F \in \mathcal{F}_{3}$, we get

$$
\sum_{F \in \mathcal{F}_{3}} \sum_{i=1}^{\mu} \sum_{j=0}^{\log m} \sum_{r=1}^{k-1} \tau(F, i, j, r) \geq\left|\mathcal{F}_{3}\right| \cdot \Omega(m) .
$$

We also compute an upper bound for the quantity on the left side of inequality (2). First, we give an upper bound on the number of triples $(Q, R, u) \in T(F, i, j, r)$ for all $F \in \mathcal{F}_{3}$. Recall that $(Q, R, u) \in T(F, i, j, r)$ implies that $Q \in \mathcal{C}_{i}$, and $R$ is an $r$-flat incident to $\ell=\Omega\left(m 2^{j-i k}\right)$ points of $P \cap Q$. Every cube $Q \in \mathcal{C}_{i}$ has volume $n / 2^{i d}$ and $P \cap Q$ is well separated. By our induction hypothesis, the number of $\ell$-rich $r$-flats in $P \cap Q$ is $O\left(\left(n / 2^{i d}\right)^{r+1} / \ell^{d+1}\right)$. The cube $Q^{\prime} \in C_{i-1}$ contains $\left|P \cap Q^{\prime}\right|=O\left(n / 2^{(i-1) k}\right)=O\left(n / 2^{i d}\right)$ points. So $P \cap Q^{\prime}$ contains $\left(O\left(n / 2^{i d}\right)\right)^{k-r}$ distinct $(k-r)$-element subsets. For all $Q \in \mathcal{C}_{i}$, we obtain an upper bound

$$
\begin{aligned}
& \sum_{F \in \mathcal{F}_{3}}|T(F, i, j, r)| \leq\left|\mathcal{C}_{i}\right| \cdot O\left(\frac{\left(n / 2^{i d}\right)^{r+1}}{\left(m 2^{j-i k}\right)^{d+1}}\right) \cdot O\left(\left(\frac{n}{2^{i d}}\right)^{k-r}\right), \\
& \sum_{F \in \mathcal{F}_{3}}|T(F, i, j, r)| \leq O\left(\frac{n^{k+1}}{m^{d+1}} \cdot 2^{i k-j(d+1)}\right) .
\end{aligned}
$$

After dividing by $\left(m 2^{j-i k}\right)^{k-r-1}$, we sum inequality (3) over all $i, j$, and $r$ :

$$
\begin{aligned}
\sum_{F \in \mathcal{F}_{3}} \tau(F, i, j, r) & \leq O\left(\frac{n^{k+1}}{m^{d+1}} \cdot 2^{i k-j(d+1)} \cdot\left(\frac{2^{i k}}{2^{j} m}\right)^{k-r-1}\right), \\
\sum_{r=1}^{k-1} \sum_{F \in \mathcal{F}_{3}} \tau(F, i, j, r) & \leq O\left(\frac{n^{k+1}}{m^{d+1}} \cdot 2^{i k-j(d+1)}\right),
\end{aligned}
$$




$$
\begin{aligned}
\sum_{j=0}^{\log m} \sum_{r=1}^{k-1} \sum_{F \in \mathcal{F}_{3}} \tau(F, i, j, r) & \leq O\left(\frac{n^{k+1}}{m^{d+1}} \cdot 2^{i k}\right), \\
\sum_{i=1}^{\mu} \sum_{j=0}^{\log m} \sum_{r=1}^{k-1} \sum_{F \in \mathcal{F}_{3}} \tau(F, i, j, r) & \leq O\left(\frac{n^{k+1}}{m^{d+1}} \cdot m\right) .
\end{aligned}
$$

By contrasting inequalities (2) and (4), we conclude that $\left|\mathcal{F}_{3}\right|=O\left(n^{k+1} / m^{d+1}\right)$. This completes the proof of Lemma 5.

Corollary 6. For every $d, k \in \mathbb{N}, 1 \leq k<d$, the number of incidences of points and $m$-rich $k$-flats in a homogeneous set of $n$ points in $\mathbb{R}^{d}$ is at most

$$
O\left(\frac{n^{k+1}}{m^{d}}\right) \text {. }
$$

Proof. In any homogeneous point set of size $n$ in $\mathbb{R}^{d}$, the number of incidences of points and $m$-rich $k$-flats is bounded by

$$
m f_{d, k}(P, m)+\sum_{j=m+1}^{n} f_{d, k}(P, j) \leq O\left(\frac{n^{k+1}}{m^{d}}\right)+\sum_{j=m+1}^{n} O\left(\frac{n^{k+1}}{j^{d+1}}\right) \leq O\left(\frac{n^{k+1}}{m^{d}}\right) .
$$

\section{Proof of Theorem 1}

We are given a homogeneous set $P$ of $n$ points in $d$-dimensions. We may choose an orthogonal coordinate system such that all coordinates of every point of $P$ are irrational and $P$ lies in the interior of cube $C$, whose vertices have rational coordinates. This guarantees that for any subdivision of $C$ into congruent subcubes, every point of $P$ lies in the interior of a subcube. Let $t$ denote the maximum number of distinct distances measured from a point of $P$ (including distance 0 ). There is a constant $\delta_{d}>d$ such that for every $s \in \mathbb{N}$, every hyperplane or sphere intersects the interior of at most $\delta_{d} s^{d-1}$ cubes in the subdivision of $C$ into $s^{d}$ congruent cubes. We subdivide $C$ into $s^{d}$ congruent subcubes $C_{1}, C_{2}, \ldots, C_{s^{d}}$, where

$$
s=\left\lfloor\left(\frac{n}{4 \delta_{d} t}\right)^{1 /(d-1)}\right\rfloor
$$

Let $T$ be a set of triples $(p, q, c) \in P^{3}$ such that

(i) $p \neq q$,

(ii) $p$ and $q$ lie in the same subcube $C_{i}$ for some $1 \leq i \leq s^{d}$,

(iii) $p$ and $q$ are equidistant from $c$.

All points are located on $n t$ spheres centered at the $n$ points of $P$. The cubes $C_{i}$, $1 \leq i \leq s^{d}$, subdivide each sphere into patches. Since every sphere intersects at most $\delta_{d} s^{d-1}$ subcubes $C_{i}$, there are at most $\delta_{d} n t s^{d-1}=n^{2} / 4$ patches, where each patch lies entirely in a subcube $C_{i}$. There are $n^{2}$ sphere-point incidences. The average number of 
points on a patch is at least four. If $x$ points lie on a sphere patch centered at $c$, then this patch contributes $\left(\begin{array}{l}x \\ 2\end{array}\right) 2$ ! triples $(p, q, c)$ to $T$. We conclude that the number of triples is $|T| \geq \Omega\left(n^{2}\right)$.

For every $m \in \mathbb{N}$, let $T_{m}$ denote the set of triples $(p, q, c) \in T$ such that the bisector hyperplane of the segment $p q$ is incident to at least $m$ but less than $2 m$ points of $P$. Since every bisector plane is incident to less than $n$ points, we can partition $T$ into $\log n$ subsets

$$
T=\bigcup_{j=0}^{\log n} T_{2^{j}} .
$$

There is a value $m=2^{j}$ for some $0 \leq j \leq \log n$, such that $\left|T_{m}\right| \geq|T| / \log n \geq$ $\Omega\left(n^{2} / \log n\right)$.

For a pair $(p, q) \in P^{2}, p \neq q$, all points of the set $M(p, q)=\{c \in P: \operatorname{dist}(p, c)=$ $\operatorname{dist}(q, c)\}$ lie on the bisector hyperplane of the line segment $p q$. Every bisector hyperplane intersects at most $\delta_{d} S^{d-1}$ subcubes, and in each subcube $C_{i}$ it can bisect at most $\left|C_{i} \cap P\right| / 2$ point pairs. So the number of pairs $(p, q) \in P^{2}$ bisected by the same hyperplane is at most

$$
\delta_{d} s^{d-1} \cdot O\left(\frac{n}{s^{d}}\right)=O\left(\frac{n}{s}\right) .
$$

Let $B_{m}$ denote the set of all bisector hyperplanes that bisect the pair $(p, q)$ for some $(p, q, c) \in T_{m}$. By definition, every hyperplane in $B_{m}$ is incident to at least $m$ but less than $2 m$ points of $P$. By Lemma 5, we have

$$
\left|B_{m}\right| \leq O\left(\frac{n^{d}}{m^{d+1}}\right) .
$$

We can now give an upper bound for $\left|T_{m}\right|$. In a triple $(p, q, c) \in T_{m}$, point $c$ lies on a bisector hyperplane of $B_{m}$. Each bisector hyperplane is incident to less than $2 m$ points of $P$ and bisects at most $O(n / s)$ pairs $(p, q)$. Therefore

$$
\begin{aligned}
\Omega\left(\frac{n^{2}}{\log n}\right) & \leq\left|T_{m}\right| \leq O\left(\frac{n^{d}}{m^{d+1}}\right) \cdot 2 m \cdot O\left(\frac{n}{s}\right), \\
m^{d} & \leq O\left(\frac{n^{d-1} \log n}{s}\right) \\
m & \leq O\left(\frac{n^{(d-1) / d} \log ^{1 / d} n}{s^{1 / d}}\right) .
\end{aligned}
$$

We obtain another upper bound for $\left|T_{m}\right|$ by the following argument. In a triple $(p, q, c) \in T_{m}$, both $p$ and $q$ lie in the same subcube $C_{i} \subset C$. There are $s^{d}$ subcubes, and each subcube contains $\left(O\left(n / s^{d}\right)\right)^{2} \leq O\left(n^{2} / s^{2 d}\right)$ point pairs. Hence, there are at most $s^{d} \cdot O\left(n^{2} / s^{2 d}\right)=O\left(n^{2} / s^{d}\right)$ such pairs $(p, q)$. For each pair $(p, q)$, where $(p, q, c) \in T_{m}$, there are at most $2 m$ points $c \in P$ on the bisector hyperplane of $p q$. We conclude that

$$
\Omega\left(\frac{n^{2}}{\log n}\right) \leq\left|T_{m}\right| \leq O\left(\frac{n^{2}}{s^{d}}\right) \cdot 2 m .
$$


Using the upper bound for $m$ from inequality (5), we have

$$
\begin{aligned}
s^{\left(d^{2}+1\right) / d} & \leq O\left(n^{(d-1) / d} \cdot \log ^{(d+1) / d} n\right), \\
\left(\frac{n}{t}\right)^{\left(d^{2}+1\right) / d(d-1)} & \leq O\left(n^{(d-1) / d} \cdot \log ^{(d+1) / d} n\right), \\
\Omega\left(n^{2 /(d-1)} \log ^{-(1+d) / d} n\right) & \leq t^{\left(d^{2}+1\right) / d(d-1)}, \\
\Omega\left(n^{2 d /\left(d^{2}+1\right)} \log ^{\left(1-d^{2}\right) /\left(d^{2}+1\right)} n\right) & \leq t,
\end{aligned}
$$

as required. This completes the proof of Theorem 1.

\section{Proof of Theorem 2}

Consider a homogeneous set $P$ of $n$ points in $\mathbb{R}^{3}$. Similarly to the previous section, we assume that all coordinates of every point in $P$ are irrational, and the vertices of the bounding cube $C$ have rational coordinates. Let $t$ denote the maximum number of distinct distances measured from a point of $P$ (including distance 0 ). We subdivide $C$ into $s^{3}$ congruent cubes $C_{1}, C_{2}, \ldots, C_{s^{3}}$, for

$$
s=\left\lfloor\sqrt{\frac{n}{\gamma t}}\right\rfloor,
$$

where $\gamma>0$ is a constant to be specified later.

By Theorem 3, $P \subset \mathbb{R}^{3}$ contains $\Omega\left(n^{2}\right)$ affine independent point pairs. This implies that there is a subset $P_{0} \subset P$ such that $\left|P_{0}\right| \geq \Omega(n)$ and every $c \in P_{0}$ is incident to $\Omega(n)$ distinct lines spanned by $P$. For every $c \in P_{0}$, let $P(c) \subset P \backslash\{c\}$ be a set of $\Omega(n)$ points such that the lines $c p, p \in P(c)$, are distinct. For every point $c \in P_{0}$, let $H_{c}$ be a unit sphere centered at $c$. For every $x \in \mathbb{R}^{3} \backslash\{c\}$, we denote by $\hat{x}$ the projection of $x$ to the unit sphere $H_{c}$. Points of $P(c)$ have distinct images in $H_{c}$ under this projection. The set of projection points is denoted by

$$
\hat{P}(c):=\{\hat{p}: c \in P(p)\} .
$$

We partition the unit sphere $H_{c}$ into $6 s^{2}$ convex spherical regions $S_{1}(c), S_{2}(c), \ldots$, $S_{6 s^{2}}(c)$ by $6 s-12$ circular arcs: Consider an axis-parallel cube centered at $c$ and subdivide each of its six faces into $s^{2}$ congruent squares, then project these squares to the sphere $H_{c}$ from $c$. The area of each spherical region is $\Theta\left(1 / s^{2}\right)$ and each one is contained in a disk of area $\Theta\left(1 / s^{2}\right)$. Every circle on the sphere $H_{c}$ intersects at most $O(s)$ regions. We then subdivide $\mathbb{R}^{d} \backslash\{c\}$ into $6 s^{2}$ regions $R_{i}(c), i=1,2, \ldots, 6 s^{2}$, such that

$$
R_{i}(c)=\left\{x \in \mathbb{R}^{d} \backslash\{c\}: \hat{x} \in S_{i}(c)\right\} .
$$

For every $c \in P_{0}$ and $j=1,2, \ldots, 6 s^{2}$, the region $R_{j}(c)$ contains $\left|P \cap R_{i}(c)\right|=$ $O\left(n / s^{2}\right)$ points because the region $R_{j}(c) \cap C$ can be covered by $O\left(n / s^{2}\right)$ unit cubes. Note also that every plane incident to $c$ intersects at most $O(s)$ regions $R_{j}(c)$, since every great circle of $S$ intersects at most $O(s)$ spherical regions $S_{j}$. If $F$ is a plane, then 
$\left|F \cap R_{j}(c) \cap P\right|=O\left(n^{2 / 3} / s\right)$ because $F \cap C$ can be covered by $O\left(n^{2 / 3}\right)$ unit cubes, and $\operatorname{area}\left(F \cap R_{j}(c)\right) \leq O(\operatorname{area}(F \cap C) / s)$.

For every $c \in P_{0}$, consider the at most $t$ spheres centered at $c$ that contain all points of $P(c)$. Every sphere $S$ centered at $c$ is partitioned into patches by the cubes $C_{i}, 1 \leq i \leq s^{3}$, and the regions $R_{j}(c), 1 \leq j \leq 6 s^{2}$. We can partition $C$ into the subcubes $C_{i}, 1 \leq i \leq s^{3}$, by $3(s-1)$ planes. These planes partition every sphere $S$ along 3(s-1) circles. Hence every sphere $S$ is partitioned by $O(s)$ circular arcs into $O\left(s^{2}\right)$ patches. We partition the points of $P$ lying on a patch into disjoint triples, after deleting at most two points from each patch if necessary. This produces a set $Q$ of quadruples $(p, q, r, c) \in P^{3} \times P_{0}$ such that

(i) the points $p, q$, and $r$ are in $P(c)$;

(ii) $p, q$, and $r$ lie on a sphere centered at $c$;

(iii) $p, q$, and $r$ lie in the same subcube $C_{i}$ for some $1 \leq i \leq s^{3}$;

(iv) $p, q$, and $r$ lie in the same regions $R_{j}(c)$, for some $1 \leq j \leq 6 s^{2}$;

(v) if $\left(p_{1}, q_{1}, r_{1}, c\right) \in Q$ and $\left(p_{2}, q_{2}, r_{2}, c\right) \in Q$, then $\left\{p_{1}, q_{1}, r_{1}\right\} \cap\left\{p_{2}, q_{2}, r_{2}\right\}=\emptyset$.

We give a lower bound on the number of quadruples in $Q$. Let $g(c)$ denote the number of patches on all $O(t)$ spheres centered at $c$ : We have $g(c)=O\left(t s^{2}\right)=O(n / \gamma)$. The average number of points on a patch centered at $c$ is $\Omega(\gamma n / g(c))=\Omega(\gamma)$. We choose the constant $\gamma>0$ such that a patch contains at least six points of $P(c)$ on average. If the $k$ th patch contains a set of points $G_{k}(c) \subset P(c)$, then $Q$ contains $\left\lfloor\left|G_{k}(c)\right| / 3\right\rfloor$ quadruples $(p, q, r, c)$. We conclude that the total number of quadruples is

$$
|Q|=\sum_{c \in P_{0}} \sum_{k=1}^{g(c)}\left\lfloor\frac{\left|G_{k}\right|}{3}\right\rfloor \geq \Omega\left(n \sum_{k=1}^{g(c)}\left(\left|G_{k}\right|-2\right)\right) \geq \Omega\left(n^{2}\right) .
$$

We define the multiplicity of a pair $(p, q) \in P^{2}$ as

$$
\begin{gathered}
m(p, q)=\mid\left\{c \in P_{0}: \exists r \text { such that }(p, q, r, c) \in Q \text { or }(q, r, p, c) \in Q\right. \\
\text { or }(r, p, q, c) \in Q\} \mid .
\end{gathered}
$$

We choose a parameter $m$ to be specified later, and distinguish two types of quadruples in $Q$ : A quadruple $(p, q, r, c)$ is low if at least one edge of the triangle $p q r$ has multiplicity at most $m$. A quadruple $(p, q, r, c)$ is high if the multiplicity of all three edges of $p q r$ are above $m$. Let $Q^{-}$and $Q^{+}$denote the sets of low and high quadruples, respectively. We distinguish two cases: First we consider the case that $\left|Q^{+}\right| \leq\left|Q^{-}\right|$, then we proceed with the case $\left|Q^{+}\right|>\left|Q^{-}\right|$.

Case $\left|Q^{+}\right| \leq\left|Q^{-}\right|$. There are at least $\Omega\left(n^{2}\right)$ low quadruples in $Q$. We define a set of triples

$$
T:=\left\{(p, q, c):(p, q, r, c) \in Q^{-}, m(p, q) \leq m\right\} .
$$

We have extracted $|T|=\Omega\left(n^{2}\right)$ triples from $Q^{-}$. Similarly to the previous section, we compute an upper bound on $|T|$. Every pair $(p, q)$ from a triple of $T$ lies in one of the $s^{3}$ subcubes of $C$, and for every pair $(p, q)$ there are at most $m$ centers $c$. Therefore, we have an upper bound

$$
|T| \leq s^{3}\left(O\left(\frac{n}{s^{3}}\right)\right)^{2} m=O\left(\frac{m n^{2}}{s^{3}}\right) .
$$


Comparing this upper bound with the lower bound $|T|=\Omega\left(n^{2}\right)$, we obtain

$$
\begin{aligned}
\Omega\left(s^{3}\right) & \leq m, \\
\Omega\left(\frac{n^{3 / 2}}{t^{3 / 2}}\right) & \leq m, \\
\Omega\left(\frac{n}{m^{2 / 3}}\right) & \leq t .
\end{aligned}
$$

Case $\left|Q^{+}\right|>\left|Q^{-}\right|$. At least half of the quadruples in $Q$ are high, and so $\left|Q^{+}\right| \geq \Omega\left(n^{2}\right)$.

For every $c \in P_{0}$, project the points of $P(c)$ to the sphere $H_{c}$. If $(p, q, r, c) \in Q$, then the intersection of the bisector plane of $p q$ and $H_{c}$ is the bisector (great circle) of the segment $\hat{p} \hat{q}$ in the sphere $H_{c}$. A (possibly degenerate) triangle $\hat{p} \hat{q} \hat{r}$ defines three distinct bisectors. The bisectors of a triangle $\hat{p} \hat{q} \hat{r}$ meet in two antipodal points on the sphere. The triangles that determine the same triple of bisectors are similar (the center of similarity is the intersection of the bisectors). Specifically, if the triangles $\hat{p}_{1} \hat{q}_{1} \hat{r}_{1}, \hat{p}_{2} \hat{q}_{2} \hat{r}_{2}, \ldots, \hat{p}_{\ell} \hat{q}_{\ell} \hat{r}_{\ell}$ determine the same triple of bisectors, then the points $\hat{p}_{1}, \hat{p}_{1}, \ldots, \hat{p}_{\ell}$ are collinear (the points $\hat{q}_{1}, \hat{q}_{2}, \ldots, \hat{q}_{\ell}$ and $\hat{r}_{1}, \hat{r}_{2}, \ldots, \hat{r}_{\ell}$ are also collinear). Every triple of bisectors determines a family of triangles. We define a family of quadruples to be a collection of quadruples $(p, q, r, c) \in Q^{+}$with a common center $c$ such that the triangles $\hat{p} \hat{q} \hat{r}$ form a family.

For every $c \in P_{0}$, we define a set of triangles in the sphere $H_{c}$ by

$$
T(c)=\left\{\hat{p} \hat{q} \hat{r}:(p, q, r, c) \in Q^{+}\right\} .
$$

By construction, all these triangles have pairwise disjoint vertex sets. There is a set $P_{1} \subseteq P_{0}$ of size $\Omega(n)$ such that for every $c \in P_{1}$, we have $|T(c)|=\Omega(n)$ triangles. For a point $c \in P_{1}$, let $B_{c}$ denote the set of $m$-rich planes incident to $c$. We denote by $\hat{B}_{c}$ the set of intersections of planes in $B_{c}$ and the unit sphere $H_{c}$, which are great circles on $H_{c}$. Note that the bisector of every edge $\hat{p} \hat{q}$ of a triangle of $T(c)$ is in $\hat{B}_{c}$.

For $c \in P_{1}$, we consider the partition of the sphere $H_{c}$ into $6 s^{2}$ regions $S_{j}(c), 1 \leq$ $j \leq 6 s^{2}$, defined above. Each triangle of $T(c)$ lies entirely in one of the regions. Let us denote by $T_{j}(c)$ the set of triangles of $T(c)$ in $S_{j}(c)$ for every $j=1,2, \ldots, 6 s^{2}$. Since the triangles have disjoint vertex sets, we have $\left|T_{j}(c)\right| \leq\left|P \cap R_{j}(c)\right| / 3 \leq O\left(n / s^{2}\right)=$ $O(t)$. But $\sum_{j=1}^{6 s^{2}}\left|T_{j}(c)\right|=\Omega(n)$, and so there are $\Omega\left(s^{2}\right)$ indices $j$ such that $\left|T_{j}(c)\right|=$ $\Omega\left(n / s^{2}\right)=\Omega(t)$. Vertices of similar triangles lie on three main circles. We have shown that every region $R_{j}(c)$ contains at most $O\left(n^{2 / 3} / s\right)=O\left(n^{1 / 6} t^{1 / 2}\right)$ coplanar points. Hence, there are at least $\Omega\left(t^{1 / 2} / n^{1 / 6}\right)$ families of triangles in $T_{j}(c)$. Since each such family determines three distinct bisectors of $\hat{B}(c)$, the triangles in $T_{j}(c)$ determine

$$
\Omega\left(\left(\frac{t^{1 / 2}}{n^{1 / 6}}\right)^{1 / 3}\right)=\Omega\left(\frac{t^{1 / 6}}{n^{1 / 18}}\right)
$$

distinct bisectors in $\hat{B}_{c}$. A bisector crosses at most $O(s)$ regions, and so we obtain the same bisector of $\hat{B}_{c}$ from at most $O(s)$ regions. We conclude that the number of bisectors determined by the $\Omega(n)$ triangles of $T(c)$ is

$$
\left|B_{c}\right| \geq \frac{\Omega\left(s^{2}\right)}{O(s)} \cdot \Omega\left(\frac{t^{1 / 6}}{n^{1 / 18}}\right) \geq \Omega\left(\sqrt{\frac{n}{t}} \cdot \frac{t^{1 / 6}}{n^{1 / 18}}\right)=\Omega\left(\frac{n^{4 / 9}}{t^{1 / 3}}\right) .
$$


Each of the $\Omega(n)$ points of $P_{1}$ is incident to $\Omega\left(n^{4 / 9} / t^{1 / 3}\right)$ distinct $m$-rich planes. This gives $\Omega\left(n^{13 / 9} / t^{1 / 3}\right)$ incidences on $m$-rich planes of $P$. By Corollary 6 , we have

$$
\begin{aligned}
\Omega\left(\frac{n^{13 / 9}}{t^{1 / 3}}\right) & \leq O\left(\frac{n^{3}}{m^{3}}\right), \\
m & \leq O\left(n^{14 / 27} t^{1 / 9}\right), \\
\Omega\left(\frac{m^{9}}{n^{14 / 3}}\right) & \leq t .
\end{aligned}
$$

In both cases we have derived lower bounds for $t$ in terms of $n$ and $m$. We choose $m \in \mathbb{N}$ such that we obtain the same result in both cases. By comparing inequalities (6) and (7), we have

$$
\begin{gathered}
\Omega\left(\frac{n^{3 / 2}}{t^{3 / 2}}\right) \leq m \leq O\left(n^{14 / 27} t^{1 / 9}\right), \\
\Omega\left(n^{53 / 87}\right) \leq t .
\end{gathered}
$$

The choice $m=n^{17 / 29}$ establishes inequality (8) in both cases. This completes the proof of Theorem 2.

\section{References}

1. P. K. Agarwal and B. Aronov, Counting facets and incidences, Discrete Comput. Geom. 7 (1992), 359-369.

2. B. Aronov, J. Pach, M. Sharir, and G. Tardos, Distinct distances in three and higher dimensions, Combin. Probab. Comput. 13 (2004), 283-293.

3. J. Beck, On the lattice property of the plane and some problems of Dirac, Motzkin and Erdôs, Combinatorica 3(3-4) (1983), 281-297.

4. J. Bourgain, On the dimension of kayela sets and related maximal inequalities, Geom. Funct. Anal. 9 (1999), 256-282.

5. F. R. K. Chung, The number of different distances determined by n points in the plane, J. Combin. Theory Ser. A 36 (1984), 342-354.

6. F. R. K. Chung, E. Szemerédi, and W. T. Trotter, The number of different distances determined by a set of points in the Euclidean plane, Discrete Comput. Geom. 7 (1992), 1-11.

7. K. L. Clarkson, H. Edelsbrunner, L. J. Guibas, M. Sharir, and E. Welzl, Combinatorial complexity bounds for arrangements of curves and spheres, Discrete Comput. Geom. 5 (1990), 99-160.

8. H. Edelsbrunner and M. Sharir, A hyperplane incidence problem with applications to counting distances, in Proc. SIGAL International Symposium on Algorithms (T. Asano et al., eds.), vol. 450 of LNCS, SpringerVerlag, Berlin, 1990, pp. 419-428.

9. Gy. Elekes and Cs. D. Tóth, Incidences of not-too-degenerate hyperplanes, in Proc. 21st ACM Symposium on Computational Geometry, ACM Press, New York, 2005, pp. 16-21.

10. P. Erdős, On sets of distances of $n$ points, Amer. Math. Monthly 53 (1946), 248-250.

11. P. Erdős, On some of my favourite theorems, in Combinatorics, Paul Erdós is Eighty, vol. 2 of Bolyai Society Mathematical Studies, János Bolyai Mathematical Society, Budapest, 1996, pp. 97-132.

12. S. Hofmann and A. Iosevich, Circular averages and Falconer-Erdôs distance conjecture in the plane for random metrics, Proc. Amer. Math. Soc. 133(1) (2005), 133-143.

13. A. Iosevich, Curvature, combinatorics, and the Fourier transform, Notices Amer. Math. Soc. 48 (2001), 577-583.

14. A. Iosevich, N. Katz, and S. Pedersen, Fourier basis and the Erdős distance problem, Math. Res. Lett. 6(2) (1999), 251-255. 
15. A. Iosevich and I. Łaba, Distance sets of well-distributed planar point sets, Discrete Comput. Geom. 31 (2004), 243-250.

16. N. H. Katz and T. Tao, Some connections between Falconer's distance set conjecture and sets of Furstenburg type, New York J. Math. 7 (2001), 149-187.

17. N. H. Katz and G. Tardos, A new entropy inequality for the Erdôs distance problem, in Towards a Theory of Geometric Graphs (J. Pach, ed.), vol. 342 of Contemporary Mathematics, American Mathematical Society, Providence, RI, 2004, pp. 119-126.

18. S. Konyagin and I. Łaba, Distance sets of well-distributed planar sets for polygonal norms, Israel J. Math., to appear.

19. L. Moser, On the different distances determined by $n$ points, Amer. Math. Monthly 59 (1952), 85-91.

20. J. Pach and M. Sharir, Geometric incidences, in Towards a Theory of Geometric Graphs (J. Pach, ed.), vol. 342 of Contemporary Mathematics, American Mathematical Society, Providence, RI, 2004, pp. 185223.

21. J. Solymosi and Cs. D. Tóth, Distinct distances in the plane, Discrete Comput. Geom. 25 (2001), 629-634.

22. J. Solymosi and V. Vu, Distinct distances in high dimensional homogeneous sets, in Towards a Theory of Geometric Graphs (J. Pach, ed.), vol. 342 of Contemporary Mathematics, American Mathematical Society, Providence, RI, 2004, pp. 259-268.

23. J. Solymosi and V. Vu, Near optimal bounds for the Erdős distinct distance problem in high dimensions, Combinatorica, to appear.

24. J. Spencer, E. Szemerédi, and W. T. Trotter, Unit distances in the Euclidean plane, in Graph Theory and Combinatorics (B. Bollobs, ed.), Academic Press, New York, 1984, pp. 293-303.

25. L. A. Székely, Crossing numbers and hard Erdős problems in discrete geometry, Combin. Probab. Comput. 6(3) (1997), 353-358.

26. E. Szemerédi and W. T. Trotter Jr., Extremal problems in discrete geometry, Combinatorica 3(3-4) (1983), 381-392.

Received March 21, 2005, and in revised form April 6, 2005, and October 17, 2005.

Online publication April 18, 2006. 